\title{
Selenium and tellurium content of stony meteorites by neutron activation
}

\author{
U. SCHINDEWOLF* \\ Department of Chemistry, University of Michigan, Ann Arbor, Michigan
}

(Received 14 January 1960)

\begin{abstract}
By neutron activation analysis the Se- and Te-content of four chondritic meteorites have been determined. The average content is $9.8 \pm 2.5$ and $0.61 \pm 1.7$ p.p.m., respectively. These values are lower than those already reported in the literature.
\end{abstract}

\section{INTRODUCTION}

SEvERAL authors (SuEss and UREY, 1956) have published data on the relative abundance of the chemical elements. In theoretically deriving these abundances extensive use has been made of certain systematic properties of nuclei because the necessary experimental data are lacking or unsatisfactory. Reliable experimental values of the abundance of each element are of great interest to cosmologists since the abundances of the elements are the result of cosmic events and any useful theory of the origin of the elements has to account in detail for the abundance curve.

A recent theory requires several different types of synthesizing processes in order to explain the features of the abundance curve (BurBIDGE et al., 1957; Cameros, 1957). Two of these processes are different types of neutron capture. Neutron capture on a long time-scale is thought to be responsible for the abundance peaks at $\mathrm{Y}$ (fifty neutrons), La (eighty-two neutrons) and $\mathrm{Pb}$ (126 neutrons) while neutron capture on a very short time-scale produces abundance peaks (CorYELL, 1956) at approximately $\mathrm{Br}, \mathrm{Xe}$ and $\mathrm{Pt}$.

Since the abundance of bromine, krypton, iodine and xenon are inherently difficult to determine, reliable data on the abundances of selenium and tellurium would be valuable in establishing the magnitudes of the peak yields at Br and Xe. BYERS (1938) reported a selenium content of 13 and 10 p.p.m. in the chondrites, Allegan, Michigan and Tabory, Russia, respectively. The data on tellurium are very uncertain and Suess and UREY (1956) report an interpolated value. Since the chemical and physical properties of selenium and tellurium are very similar, the abundance ratio is a more meaningful quantity than the individual absolute abundances. The Se/Te abundance ratio should thus be rather constant from one meteorite to another. GoLdschmid (1954) estimated a Se/Te atomic ratio of 80 from the data by the Nodpacks, and Sumss and URey chose a value of 14.5 while pointing out the need for additional experimental data.

The present paper reports a new determination of the concentrations of selenium and tellurium in chondrites which are supposed to contain the non-volatile elements in relative cosmic abundances. The method of neutron activation was

\footnotetext{
* Present address: Institut für Kernverfahrenstechnik, Technische Hochschule, Karlsruhe, Germany.
} 
applied which for the analysis of trace elements has many advantages over conventional analytical techniques, one of the important advantages being the elimination of reagent contamination.

\section{EXPERIMENTAL}

\section{Irradiation}

Powdered meteorite samples of from 50 to $200 \mathrm{mg}$ were packaged in aluminium foil and irradiated with various neutron monitors in the Argonne CP-5 heavy-water reactor. The selenium and tellurium content of the aluminium foil was low enough so that contamination from the wrapping material was negligible.

Two types of flux monitors were employed. One group was prepared by introducing a known amount of natural selenium or tellurium (an amount at least fifty times the original content) into a meteorite matrix. These monitors were chemically processed in a manner similar to the samples of unknown selenium and tellurium content. The other group of flux monitors was prepared by evaporating a known amount $(5-10 \mu \mathrm{g})$ of selenium or tellurium in solution onto filter papers which were wrapped in aluminium foil before irradiation. Both monitors gave identical results for selenium and tellurium, indicating that neutron attenuation in the meteoritic material does not affect the results of the activation analysis.

The selenium content in the meteorites was determined by neutron capture of $\mathrm{Se}^{74}(0.87$ atom per cent in normal selenium; neutron capture cross-section of 26 barns). The neutron capture product is $128-d \mathrm{Se}^{75}$ which is easily detected and identified by three intense $\gamma$-rays of 136,267 and $402 \mathrm{keV}$. With suitable irradiation and decay periods, the $\gamma$-rays associated with selenium activities produced by neutron capture on the other stable isotopes of selenium do not interfere with a quantitative measurement of the intensity of the $\gamma$-rays of Se ${ }^{75}$. Even though a shorter irradiation time and a greater sensitivity (SCHINDEWOLF, 1958) of selenium can be achieved by measuring the radiations of other selenium isotopes produced by neutron activation, $\mathrm{Se}^{75}$ is the most convenient isotope for the neutron activation analysis of the element. The meteorites were irradiated for about 1 week prior to the selenium analysis.

The tellurium content of the meteorites was determined from the $\beta$-activity of the $9.4 \mathrm{hr} \mathrm{Te}^{127}$ formed with a 0.8 barn cross-section by neutron capture on $\mathrm{Te}^{126}$ (18.7 atom per cent in normal tellurium). Shorter-lived tellurium isotopes produced by neutron capture were not considered because they necessitated very rapid chemical separation, and the longer-lived isotopes were excluded since they are less sensitive than $\mathrm{Te}^{127}$ for tellurium analysis by neutron activation. The meteorite samples were irradiated for about $12 \mathrm{hr}$ for the tellurium analyses.

\section{Chemical separation}

The chemistry of selenium and tellurium is very similar and the same procedures were used initially in both analyses to separate these elements from the remainder of the sample. After neutron irradiation and a suitable decay time, about $20 \mathrm{mg}$ of either selenium or tellurium and 1 to $2 \mathrm{~g}$ of sodium peroxide were added to the powdered meteorite and fused in a nickel orucible. After a cooling period, 
the solid cake was dissolved in $6 \mathrm{~N} \mathrm{HCl}$. Selenium and tellurium were then precipitated as the element from a boiling solution by reduction with $\mathrm{SO}_{2}$ gas. Further purification was achieved by a modification of the procedure by GLENDENIN (Coryell and Sugerman, 1951). Selenium and tellurium were dissolved in aquaregia, the solution was made basic with $\mathrm{NH}_{4} \mathrm{OH}$, and ferric iron added as a scavenging agent for impurities which coprecipitate with $\mathrm{Fe}(\mathrm{OH})_{3}$.

A separation of selenium from tellurium was achieved on a Dowex-1 (100-200 mesh) anion exchange column which was $0.8 \mathrm{~cm}$ in diameter and $4 \mathrm{~cm}$ in length (Schindewolf, 1957). Selenium was eluted from the column with from three to five column volumes of $3 \mathrm{~N} \mathrm{HCl}$, while tellurium is strongly adsorbed (distribution coefficient of $10^{3}$ ). Such a procedure can give a separation factor of selenium from tellurium of greater than $10^{4}$. Tellurium is then eluted rapidly with $0 \cdot 2-0 \cdot 5 \mathrm{~N} \mathrm{HCl}$. The selenium and tellurium samples were prepared for counting by another precipitation with $\mathrm{SO}_{2}$, filtration and mounting. Chemical yields in all experiments ranged between 50 and 80 per cent. The efficiency of the chemical separation procedure was tested with radioactive tracers of a number of typical elements such as $\mathrm{Ag}$, Ce, $\mathrm{Co}, \mathrm{Cs}, \mathrm{Hg}, \mathrm{Ir}, \mathrm{Nb}, \mathrm{Ru}, \mathrm{Sb}, \mathrm{Sc}, \mathrm{Ta}, \mathrm{Zn}$ and $\mathrm{Zr}$. Decontamination factors of greater than $10^{4}$ were found for all these elements.

\section{Counting techniques}

The selenium $\gamma$-rays were detected and analysed with a 3 in. by 3 in. $\mathrm{NaI}(\mathrm{Tl})$ scintillation crystal coupled to a 256-channel spectrometer. The quantitative analysis for selenium in each of the various meteorites was made by comparing the $\mathrm{Se}^{75} \gamma$-ray activity of each of the irradiated meteorites with the correspondingly irradiated selenium monitor. Their spectra were indistinguishable except for intensity and no $\gamma$-rays due to contaminating activities were detected. Decay measurements of the $\gamma$-rays from the meteorite selenium and the flux monitor selenium gave the same half-life. With our experimental conditions approximately $2 \times 10^{4} \gamma$-ray counts $/$ min of $\mathrm{Se}^{75}$ were observed per $\mu \mathrm{g}$ of selenium.

The $9 \cdot 4$-hr $\mathrm{Te}^{127} \beta$-activity was counted with an end-window proportional counter. Tellurium (1 $\mu \mathrm{g}$ ) gave approximately $10^{5} \mathrm{Te}^{127} \beta$-counts $/ \mathrm{min}$. The decay of the tellurium samples from the meteorites and of the tellurium flux monitors was followed for $24 \mathrm{hr}$. The decay curves of the meteorite tellurium and the flux monitor tellurium were parallel in all cases except for one Holbrook sample. $\gamma$-Ray analysis of this sample revealed the presence of $77-\mathrm{hr} \mathrm{Te}^{132}$ with its $2 \cdot 3$-hr I $\mathrm{I}^{132}$ daughter. The latter tellurium isotope resulted from the fission of uranium with which this Holbrook sample was contaminated. An estimate of the amount of $9 \cdot 4-\mathrm{hr} \mathrm{Te}^{127}$ for this meteorite was made by an analysis of the $\beta$-decay curves. The $\mathrm{Te}^{127}$ is assumed to result only from the neutron activation of $\mathrm{Te}^{126}$. This is a reasonable assumption because the fission yield of the 127-mass chain is much less than the 132-mass chain and besides this yield is held up at the 93-hr $\mathrm{Sb}^{127}$.

An attempt was made to separate the tellurium from the meteorite before irradiation by first dissolving the meteorite in a $\mathrm{HClO}_{4}-\mathrm{HF}$ solution and then coprecipitating the tellurium with highly-purified selenium. This experiment failed due to substantial loss of tellurium even though the coprecipitation is known 
to be almost complete. The tellurium was probably lost as volatile $\mathrm{TeF}_{4}$ and/or $\mathrm{TeF}_{6}$ during the dissolution with HF.

\section{Results and Discussion}

The results of the Se and Te activation analysis for several of the stony meteorites are listed in Table 1.

Table 1. Selenium and tellurium content of meteorites

\begin{tabular}{l|c|c|c}
\hline Meteorite & $\begin{array}{c}\text { Selenium } \\
\text { (p.p.m. by weight) }\end{array}$ & $\begin{array}{c}\text { Tellurium } \\
\text { (p.p.m. by weight) }\end{array}$ & $\begin{array}{c}\text { Se/Te } \\
\text { (weight ratio) }\end{array}$ \\
\hline Chondrites & & & \\
Beardsley & $10 \cdot 8$ & $0 \cdot 88$ & $12 \cdot 3$ \\
Forest City & $8 \cdot 6$ & $0 \cdot 46$ & $18 \cdot 7$ \\
Holbrook & $13 \cdot 3$ & $0 \cdot 62$ & $21 \cdot 4$ \\
Modoc & $6 \cdot 5$ & $0 \cdot 46$ & $14 \cdot 1$ \\
Achondrites & $0 \cdot 007$ & $0 \cdot 007$ & \\
Johnstown, Colo. & $0 \cdot 0016$ & & \\
Nuevo Laredo & $5 \cdot 8$ & & \\
Brenham, Kansas & & & \\
\hline
\end{tabular}

Each entry in Table 1 is an average of two independent determinations and is believed to be accurate to within about 15 per cent except the tellurium content of Holbrook where an error of 50 per cent is likely. The values of selenium and tellurium in Johnstown, Colo., are upper limits. The average selenium and tellurium contents of the chondritic meteorites Beardsley, Forest City, Holbrook and Modoc are $9.8 \pm 2.5$ p.p.m. and $0.61 \pm 0.17$ p.p.m., respectively. The atomic abundances of these elements relative to silicon $=10^{6}$ have been calculated and are listed in Table 2 along with values derived by other authors. The relative weight of silicon in primitive solar non-volatile material is taken as 18.5 per cent.

Table 2. Cosmic atomic abundances of selenium and tellurium

\begin{tabular}{|c|c|c|c|c|}
\hline & GOLDSCHMIDT & UREY & Suess and UREY & This paper \\
\hline $\begin{array}{l}\mathrm{Se} \\
\mathrm{Te}\end{array}$ & $13 \cdot 5$ & 24 & $67 \cdot 6$ & $\begin{array}{c}18.8 \\
0.73\end{array}$ \\
\hline $\mathrm{Se} / \mathrm{Te}$ & 80 & 150 & $14 \cdot 5$ & $25 \cdot 8$ \\
\hline
\end{tabular}

The data on the Se- and Te-content of chondritic meteorites presented here are lower than previously assumed and indicate that the abundance peaks around the elements $\mathrm{Br}$ and $\mathrm{Xe}$ which are caused by neutron-capture processes on a short time scale, are lower than reported. It would be too hasty, however, to reconstruct the abundance curve without a better knowledge of the abundance of neighbouring elements such as $\mathrm{As}, \mathrm{Rb}$ and $\mathrm{Sr}$, and $\mathrm{Sb}, \mathrm{Cs}$ and $\mathrm{Ba}$. The abundance of the elements $\mathrm{Br}$ and $\mathrm{Kr}$ as well as $\mathrm{I}$ and $\mathrm{Xe}$ can be obtained by interpolation only because, owing to their volatility, no reliable data for these elements will be available from analysis of meteorites. It therefore seems necessary to wait for new analytical data of the $\mathrm{As}, \mathrm{Rb}, \mathrm{Sr}, \mathrm{Sb}, \mathrm{Cs}$ and $\mathrm{Ba}$ content of meteorites. 
Acknowledgements - The writer is grateful to Drs. W. M. Manning and J. R. Huizinga for the permission to carry out the investigation in the Chemistry Division of the Argonne National Laboratory and to the staff of the Argonne CP-5 heavy-water reactor for the irradiations. He is also indebted to Profs. H. C. UReY, H. E. Sukss, J. R. Hulzenga and W. W. Meinke for valuable discussions and encouragement. This work was supported in part by the U.S. Atomic Energy Commission.

\section{REFERENCES}

Burbidge E. M., Burbidge G. R., Fowler W. A. and Hoyle F. (1957) Rev. Mod. Phys. 29, 547.

Byers H. (1938) Industr. Engng. Chem. (News) 16, 459.

Cameron A. G. W. (1957) Canadian Atomic Energy Report AECL-454.

Coryell C. D. (1956) Annual Report No. 53, Massachusette Institute of Technology Laboratory for Nuclear Science (1956).

Coryell C. D. and Sugarmay N. (1951) Radiochemical Studies. Fission Products. National Nuclear Energy Series IV, Vol. 9, p. 596. McGraw-Hill, New York.

Goldschmid V. M. (1954) Geochemistry. Clarendon Press, Oxford.

SCHINDEwoLT U. (1957) Z. angew. Chem. 69, 226.

SCHINDEWOLF U. (1958) Z. angew. Chem. 70, 181.

Suess H. E. and UReY H. C. (1956) Rev. Mod. Phys. 28, 53. 